\title{
The Hermite-Hadamard inequality for $r$-convex functions
}

\author{
Gholamreza Zabandan', Abasalt Bodaghi² and Adem Kılıçman ${ }^{3 *}$
}

${ }^{\text {*Correspondence: }}$

akilicman@putra.upm.edu.my

${ }^{3}$ Department of Mathematics and Institute for Mathematical Research, University Putra Malaysia, UPM, Serdang, Selangor 43400, Malaysia Full list of author information is available at the end of the article

\begin{abstract}
In this paper, we establish the Hermite-Hadamard inequality for $r$-convex functions. We prove that $r$-convexity implies $s$-convexity $(0 \leq r \leq s)$. As a result, we obtain a refinement of the Hermite-Hadamard inequality for an $r$-convex function $(0 \leq r \leq 1)$. We also investigate the Hermite-Hadamard inequality for the product of an $r$-convex function $f$ and an s-convex function $g$.
\end{abstract}

MSC: $26 \mathrm{D} 15 ; 26 \mathrm{D} 10$

Keywords: Hermite-Hadamard inequality; integral inequality

\section{Introduction}

Let $f:[a, b] \longrightarrow R$ be a convex function, then the inequality

$$
f\left(\frac{a+b}{2}\right) \leq \frac{1}{b-a} \int_{a}^{b} f(x) d x \leq \frac{f(a)+f(b)}{2}
$$

is known as the Hermite-Hadamard inequality (see [1] for more information). Since then, some refinements of the Hermite-Hadamard inequality on convex functions have been extensively investigated by a number of authors (e.g., [2, 3] and [4]). In [5], the first author obtained a new refinement of the Hermite-Hadamard inequality for convex functions. The Hermite-Hadamard inequality was generalized in [6] to an $r$-convex positive function which is defined on an interval $[a, b]$. A positive function $f$ is called $r$-convex on $[a, b]$, if for each $x, y \in[a, b]$ and $t \in[0,1]$,

$$
f(t x+(1-t) y) \leq \begin{cases}{\left[t f^{r}(x)+(1-t) f^{r}(y)\right]^{\frac{1}{r}},} & r \neq 0, \\ {[f(x)]^{t}[f(y)]^{1-t},} & r=0 .\end{cases}
$$

It is obvious 0 -convex functions are simply log-convex functions and 1-convex functions are ordinary convex functions. One should note that if $f$ is $r$-convex in $[a, b]$, then $f^{r}$ is a convex function $(r>0)$.

Some refinements of the Hadamard inequality for $r$-convex functions could be found in [7] and [8]. In [9], Bessenyei studied Hermite-Hadamard-type inequalities for generalized 3-convex functions. In [7], the authors showed that if $f$ is $r$-convex in $[a, b]$ and $0<r \leq 1$, then

$$
\frac{1}{b-a} \int_{a}^{b} f(x) d x \leq \frac{r}{r+1}\left[f^{r}(a)+f^{r}(b)\right]^{\frac{1}{r}} .
$$

(C) 2012 Zabandan et al.; licensee Springer. This is an Open Access article distributed under the terms of the Creative Commons Attribution License (http://creativecommons.org/licenses/by/2.0), which permits unrestricted use, distribution, and reproduction in any medium, provided the original work is properly cited. 
In this paper, first we show that if $f$ is $r$-convex in $[a, b]$ and $r \geq 1$, then

$$
\frac{1}{b-a} \int_{a}^{b} f(x) d x \leq\left[\frac{1}{2}\left(f^{r}(a)+f^{r}(b)\right)\right]^{\frac{1}{r}} .
$$

In Theorem 2.3, we prove the following inequality for $r$-convex functions:

$$
\frac{1}{b-a} \int_{a}^{b} f(x) d x \leq \frac{r}{r+1} \cdot \frac{f^{r+1}(b)-f^{r+1}(a)}{f^{r}(b)-f^{r}(a)} \quad(r>0) .
$$

The inequality (4) is an extension and refinement of (2) and (3). In Theorem 2.4, we show that $r$-convexity implies $s$-convexity $(0 \leq r \leq s)$. We employ this result in Theorem 2.6 and Corollary 2.7 to refine the Hermite-Hadamard inequality by $r$-convexity $(0 \leq r \leq 1)$. Finally, we generalize some results in [7] without using Minkowski's inequality. Indeed, we obtain refinements for the product of an $r$-convex function $f$ and an $s$-convex function $g$ $(r, s \geq 0)$.

\section{Main results}

Theorem 2.1 Let $f:[a, b] \longrightarrow(0, \infty)$ be r-convex and $r \geq 1$. Then the following inequality holds:

$$
\frac{1}{b-a} \int_{a}^{b} f(x) d x \leq \frac{1}{2}\left[\left(f^{r}(a)+f^{r}(b)\right)\right]^{\frac{1}{r}} .
$$

Proof Since $r \geq 1$, by Jensen's inequality, we have

$$
\left(\frac{1}{b-a} \int_{a}^{b} f(x) d x\right)^{r} \leq \frac{1}{b-a} \int_{a}^{b} f^{r}(x) d x .
$$

By convexity of $f^{r}$ and the right side of (1), we obtain

$$
\frac{1}{b-a} \int_{a}^{b} f^{r}(x) d x \leq \frac{1}{2}\left(f^{r}(a)+f^{r}(b)\right) .
$$

Thus,

$$
\frac{1}{b-a} \int_{a}^{b} f(x) d x \leq\left[\frac{1}{2}\left(f^{r}(a)+f^{r}(b)\right)\right]^{r}
$$

Corollary 2.2 Let $f:[a, b] \longrightarrow(0, \infty)$ be a 1 -convex function. Then

$$
\frac{1}{b-a} \int_{a}^{b} f(x) d x \leq \frac{1}{2}(f(b)+f(a)) .
$$

Theorem 2.3 Let $f:[a, b] \longrightarrow(0, \infty)$ be $r$-convex and $r \geq 0$. Then the following inequalities hold:

$$
\frac{1}{b-a} \int_{a}^{b} f(x) d x \leq \begin{cases}\frac{r}{r+1}\left(\frac{f^{r+1}(b)-f^{r+1}(a)}{f^{r}(b)-f^{r}(a)}\right), & r \neq 0, \\ {[f(b)-f(a)] \ln \frac{f(b)}{f(a)},} & r=0 .\end{cases}
$$


Proof First, let $r>0$. Since $f$ is $r$-convex, for all $t \in[0,1]$, we have

$$
f(t a+(1-t) b) \leq\left[t f^{r}(a)+(1-t) f^{r}(b)\right]^{\frac{1}{r}} .
$$

It is easy to observe that

$$
\begin{aligned}
\frac{1}{b-a} \int_{a}^{b} f(x) d x & =\int_{0}^{1} f(t a+(1-t) b) d t \\
& \leq \int_{0}^{1}\left[t f^{r}(a)+(1-t) f^{r}(b)\right]^{\frac{1}{r}} d t \\
& =\int_{0}^{1}\left[t\left(f^{r}(a)-f^{r}(b)\right)+f^{r}(b)\right]^{\frac{1}{r}} d t .
\end{aligned}
$$

By substitution $t\left(f^{r}(a)-f^{r}(b)\right)+f^{r}(b)=z$, we obtain

$$
\begin{aligned}
\frac{1}{b-a} \int_{a}^{b} f(x) d x & \leq \frac{1}{f^{r}(b)-f^{r}(a)} \int_{f^{r}(a)}^{f^{r}(b)} z^{\frac{1}{r}} d z \\
& =\frac{1}{f^{r}(b)-f^{r}(a)} \cdot \frac{1}{1+\frac{1}{r}}\left[z^{1+\frac{1}{r}}\right]_{f^{r}(a)}^{f^{r}(b)} \\
& =\frac{r}{r+1}\left(\frac{f^{r+1}(b)-f^{r+1}(a)}{f^{r}(b)-f^{r}(a)}\right)
\end{aligned}
$$

For $r=0$, we have

$$
f(t x+(1-t) y) \leq[f(x)]^{t}[f(y)]^{1-t} .
$$

So,

$$
\begin{aligned}
\frac{1}{b-a} \int_{a}^{b} f(x) d x & =\int_{0}^{1} f(t a+(1-t) b) d t \\
& \leq \int_{0}^{1}[f(a)]^{t}[f(b)]^{1-t} d t \\
& =f(b) \int_{0}^{1}\left[\frac{f(a)}{f(b)}\right]^{t} d t \\
& =\left.f(b)\left[\frac{f(a)}{f(b)}\right]^{t} \ln \frac{f(a)}{f(b)}\right|_{0} ^{1} \\
& =[f(b)-f(a)] \ln \frac{f(b)}{f(a)} .
\end{aligned}
$$

The proof is completed.

With the hypotheses of Theorem 2.3, if $f(a)=f(b)$, its proving process shows that $\frac{1}{b-a} \int_{a}^{b} f(x) d x$ can be dominated by $f(a)$ where $r \geq 0$.

Note that if we put $r=1$ in Theorem 2.3, we can obtain again the inequality (5).

Theorem 2.4 Let $f:[a, b] \longrightarrow(0, \infty)$ be r-convex on $[a, b]$ and $0 \leq r \leq s$. Then $f$ is $s$-convex. In particular, iff is $r$-convex and $0 \leq r \leq 1$, then $f$ is convex. 
In order to prove the above theorem, we need the following lemma.

Lemma 2.5 If $0 \leq \alpha \leq 1$ and $0 \leq r \leq s$, then the following inequalities hold for every pair of non-negative real numbers $x$ and $y$ :

$$
x^{\alpha} y^{1-\alpha} \leq\left(\alpha x^{r}+(1-\alpha) y^{r}\right)^{\frac{1}{r}} \leq\left(\alpha x^{s}+(1-\alpha) y^{s}\right)^{\frac{1}{s}} .
$$

Proof The left side of the inequality is clear by Young's inequality. The right side is obvious if either $x$ or $y$ equals zero. So, let $x>0$ and $y>0$. Consider $f:[0, \infty) \rightarrow R$ defined by

$$
f(t)=\left(\alpha t^{r}+1-\alpha\right)^{s}-\left(\alpha t^{s}+1-\alpha\right)^{r} .
$$

Then $f^{\prime}(t)=r s \alpha\left[t^{r-1}\left(\alpha t^{r}+1-\alpha\right)^{s-1}-t^{s-1}\left(\alpha t^{s}+1-\alpha\right)^{r-1}\right]$. So, $t=1$ is a critical point of $f$. By an easy calculation, we see that $f^{\prime \prime}(1)=r s \alpha(1-\alpha)(r-s) \leq 0$. It follows that $f$ attains its maximum at $t=1$. Thus, $f(t) \leq f(1)=0$. This shows that

$$
\left(\alpha t^{r}+1-\alpha\right)^{s} \leq\left(\alpha t^{s}+1-\alpha\right)^{r}
$$

Now, if we put $t=\frac{x}{y}$ in the above inequality, we get

$$
\left(\alpha x^{r}+(1-\alpha) y^{r}\right)^{s} \leq\left(\alpha x^{s}+(1-\alpha) y^{s}\right)^{r}
$$

Therefore, we can deduce the right side of (6) by taking $r$ sth root.

Proof of Theorem 2.4 Since $f$ is $r$-convex, by Lemma 2.5 for all $x, y \in[a, b]$ and $t \in[0,1]$, we have

$$
f(t x+(1-t) y) \leq \begin{cases}t\left[f^{r}(x)+(1-t) f^{r}(y)\right]^{\frac{1}{r}} \leq\left[t f^{s}(x)+(1-t) f^{s}(y)\right]^{\frac{1}{s}}, & 0<r \leq s \\ {[f(x)]^{t}[f(y)]^{1-t} \leq\left[t f^{s}(x)+(1-t) f^{s}(y)\right]^{\frac{1}{s}},} & 0=r \leq s .\end{cases}
$$

Hence, $f$ is $s$-convex.

Theorem 2.6 Let $f:[a, b] \longrightarrow(0, \infty)$ be $r$-convex on $[a, b]$ and $0 \leq r \leq s$. Then the following inequalities hold:

$$
\frac{1}{b-a} \int_{a}^{b} f(x) d x \leq \begin{cases}\frac{r}{r+1} \frac{f^{r+1}(b)-f^{r+1}(a)}{f^{r}(b)-f^{r}(a)} \leq \frac{s}{s+1}\left(\frac{f^{s+1}(b)-f^{s+1}(a)}{f^{s}(b)-f^{s}(a)}\right), & 0<r \leq s \\ {[f(b)-f(a)] \ln \frac{f(b)}{f(a)} \leq \frac{s}{s+1}\left(\frac{f^{s+1}(b)-f^{s+1}(a)}{f^{s}(b)-f^{s}(a)}\right),} & 0=r<s .\end{cases}
$$

Proof The left side of the inequalities is clear by Theorem 2.3. For the right side, by the inequality in (6), we have

$$
\left[t f^{r}(x)+(1-t) f^{r}(b)\right]^{\frac{1}{r}} \leq\left[t f^{s}(a)+(1-t) f^{s}(b)\right]^{\frac{1}{s}} .
$$

By integrating it on $[0,1]$, we obtain

$$
\int_{0}^{1}\left[t f^{r}(a)+(1-t) f^{r}(b)\right] d t \leq \int_{0}^{1}\left[t f^{s}(a)+(1-t) f^{s}(b)\right]^{\frac{1}{t}} d t .
$$


Thus,

$$
\frac{r}{r+1}\left(\frac{f^{r+1}(b)-f^{r+1}(a)}{f(b)-f(a)}\right) \leq \frac{s}{s+1}\left(\frac{f^{s+1}(b)-f^{s+1}(a)}{f^{s}(b)-f^{s}(a)}\right) .
$$

Also, another inequality can be deduced by integrating the inequalities in (6) if we replace $x$ and $y$ by $f(x)$ and $f(y)$, respectively.

Corollary 2.7 Let $f:[a, b] \longrightarrow(0, \infty)$ be r-convex and $0 \leq r \leq 1$. Then

$$
\begin{aligned}
f\left(\frac{a+b}{2}\right) & \leq \frac{1}{b-a} \int_{a}^{b} f(x) d x \leq[f(b)-f(a)] \ln \frac{f(b)}{f(a)} \\
& \leq \frac{r}{r+1}\left(\frac{f^{r+1}(b)-f^{r+1}(a)}{f^{r}(b)-f^{r}(a)}\right) \leq \frac{f(a)+f(b)}{2} .
\end{aligned}
$$

In other words, when $f$ is $r$-convex and $0 \leq r \leq 1$, we can refine the Hermite-Hadamard inequalities through Theorem 2.6.

Theorem 2.8 Let $f, g:[a, b] \longrightarrow(0, \infty)$ be r-convex and s-convex functions respectively on $[a, b]$ and $r, s>0$. Then the following inequality holds:

$$
\begin{aligned}
\frac{1}{b-a} \int_{a}^{b} f(x) g(x) d x \leq & \frac{1}{2}\left(\frac{r}{r+2}\right)\left(\frac{f^{r+2}(b)-f^{r+2}(a)}{f^{r}(b)-f^{r}(a)}\right) \\
& +\frac{1}{2}\left(\frac{s}{s+2}\right)\left(\frac{g^{s+2}(b)-g^{s+2}(a)}{g^{s}(b)-g^{s}(a)}\right) \\
& (f(a) \neq f(b), g(a) \neq g(b)) .
\end{aligned}
$$

Proof Since $f$ is $r$-convex and $g$ is $s$-convex, for all $t \in[0,1]$, we have

$$
\begin{aligned}
& f(t a+(1-t) b) \leq\left[t f^{r}(a)+(1-t) f^{r}(b)\right]^{\frac{1}{r}}, \\
& g(t a+(1-t) b) \leq\left[\operatorname{tg}^{s}(a)+(1-t) g^{s}(b)\right]^{\frac{1}{s}} .
\end{aligned}
$$

Thus,

$$
\begin{aligned}
\frac{1}{b-a} \int_{a}^{b} f(x) g(x) d x & =\int_{0}^{1} f(t a+(1-t) b) g(t a+(1-t) b) d t \\
& \leq \int_{0}^{1}\left[t f^{r}(a)+(1-t) f^{r}(b)\right]^{\frac{1}{r}}\left[\operatorname{tg}^{s}(a)+(1-t) g^{s}(b)\right]^{\frac{1}{s}} d t \\
& =\int_{0}^{1}\left[t\left(f^{r}(a)-f^{r}(b)\right)+f^{r}(b)\right]^{\frac{1}{r}}\left[t\left(g^{s}(a)-g^{s}(b)\right)+g^{s}(b)\right]^{\frac{1}{s}} d t .
\end{aligned}
$$

Applying Cauchy's inequality, we get

$$
\begin{aligned}
& \int_{0}^{1}\left[t\left(f^{r}(a)-f^{r}(b)\right)+f^{r}(b)\right]^{\frac{1}{r}}\left[t\left(g^{s}(a)-g^{s}(b)\right)+g^{s}(b)\right]^{\frac{1}{s}} d t \\
& \quad \leq \frac{1}{2} \int_{0}^{1}\left[t\left(f^{r}(a)-f^{r}(b)\right)+f^{r}(b)\right]^{\frac{2}{r}} d t+\frac{1}{2} \int_{0}^{1}\left[t\left(g^{s}(a)-g^{s}(b)\right)+g^{s}(b)\right]^{\frac{2}{s}} d t .
\end{aligned}
$$


Similar to the proof of Theorem 2.3 and by substitution $t\left(f^{r}(a)-f^{r}(b)\right)+f^{r}(b)=z$, we obtain

$$
\int_{0}^{1}\left[t\left(f^{r}(a)-f^{r}(b)\right)+f^{r}(b)\right]^{\frac{2}{r}} d t=\frac{r}{r+2}\left(\frac{f^{r+2}(b)-f^{r+2}(a)}{f^{r}(b)-f^{r}(a)}\right) .
$$

Similarly,

$$
\int_{0}^{1}\left[t\left(g^{s}(a)-g^{s}(b)\right)+g^{s}(b)\right]^{\frac{1}{s}} d t=\frac{s}{s+2}\left(\frac{g^{s+2}(b)-g^{s+2}(a)}{g^{s}(b)-g^{s}(a)}\right) .
$$

Using (7), (8) and (9), we can obtain the desired result.

Remark 2.9 If the conditions of Theorem 2.8 hold, and $r \leq s$, by Theorem 2.4, $f$ is $s$-convex. Thus, the result of Theorem 2.8 could be as follows:

$$
\frac{1}{b-a} \int_{a}^{b} f(x) g(x) d x \leq \frac{1}{2}\left(\frac{s}{s+2}\right)\left[\frac{f^{s+2}(b)-f^{s+2}(a)}{f^{s}(b)-f^{s}(a)}+\frac{g^{s+2}(b)-g^{s+2}(a)}{g^{s}(b)-g^{s}(a)}\right] .
$$

If $f=g$, we have

$$
\frac{1}{b-a} \int_{a}^{b} f^{2}(x) d x \leq \frac{s}{s+2}\left(\frac{f^{s+2}(b)-f^{s+2}(a)}{f^{s}(b)-f^{s}(a)}\right) .
$$

Now, if $f=g$ and $r=s=2$ in Theorem 2.8 , we have

$$
\frac{1}{b-a} \int_{a}^{b} f^{2}(x) d x \leq \frac{1}{2}\left(f^{2}(b)+f^{2}(a)\right)
$$

which is the same result as in [7, Corollary 2.5]. This shows that Theorem 2.8 is a generalization of [7, Theorem 2.3]. In fact, the condition $r, s \leq 2$ is redundant.

Theorem 2.10 Let $f, g:[a, b] \longrightarrow(0, \infty)$ be 0 -convex on $[a, b]$. Then the following inequality holds:

$$
\frac{1}{b-a} \int_{a}^{b} f(x) g(x) d x \leq[f(b) g(b)-f(a) g(a)] \ln \frac{f(b) g(b)}{f(a) g(a)}
$$

Proof Since $f$ and $g$ are 0 -convex, for all $t \in[0,1]$, we have

$$
\begin{aligned}
& f(t x+(1-t) y) \leq[f(x)]^{t}[f(y)]^{1-t}, \\
& g(t x+(1-t) y) \leq[g(x)]^{t}[g(y)]^{1-t} .
\end{aligned}
$$

For all $x, y \in[0,1]$, and thus

$$
\begin{aligned}
\frac{1}{b-a} \int_{a}^{b} f(x) g(x) d x & =\int_{0}^{1}[f(t a+(1-t) b)][g(t x+(1-t) y)] d t \\
& \leq \int_{0}^{1}[f(a)]^{t}[f(b)]^{1-t}[g(a)]^{t}[g(b)]^{1-t} d t
\end{aligned}
$$




$$
\begin{aligned}
& =f(b) g(b) \int_{0}^{1}\left[\frac{f(a) g(a)}{f(b) g(b)}\right]^{t} d t \\
& =[f(b) g(b)-f(a) g(a)] \ln \frac{f(b) g(b)}{f(a) g(a)} .
\end{aligned}
$$

Corollary 2.11 With the hypotheses of the above theorem and $f=g$, we have

$$
\frac{1}{b-a} \int_{a}^{b} f^{2}(x) d x \leq\left[f^{2}(b)-f^{2}(a)\right] \ln \frac{f^{2}(b)}{f^{2}(a)} .
$$

Theorem 2.12 Let $f, g:[a, b] \longrightarrow(0, \infty)$ be $r$-convex and 0 -convex functions respectively on $[a, b]$ and $r>0$. Then the following inequality holds:

$$
\begin{aligned}
& \frac{1}{b-a} \int_{a}^{b} f(x) g(x) d x \leq \frac{1}{2}\left(\frac{r}{r+2}\right)\left(\frac{f^{r+2}(b)-f^{r+2}(a)}{f^{r}(b)-f^{r}(a)}\right)+\frac{1}{4}\left[g(b)^{2}-g(a)^{2}\right] \ln \frac{g(b)}{g(a)} \\
& \quad(f(a) \neq f(b)) .
\end{aligned}
$$

Proof Since $f$ is $r$-convex and $g$ is 0 -convex, for all $t \in[0,1]$, we have

$$
\begin{aligned}
& f(t a+(1-t) b) \leq\left[t f^{r}(a)+(1-t) f^{r}(b)\right]^{\frac{1}{r}}, \\
& g(t x+(1-t) y) \leq[g(x)]^{t}[g(y)]^{1-t} .
\end{aligned}
$$

Thus,

$$
\begin{aligned}
\frac{1}{b-a} \int_{a}^{b} f(x) g(x) d x & =\int_{0}^{1} f(t a+(1-t) b) g(t a+(1-t) b) d t \\
& \leq \int_{0}^{1}\left[t f^{r}(a)+(1-t) f^{r}(b)\right]^{\frac{1}{r}}[g(a)]^{t}[g(b)]^{1-t} d t \\
& =\int_{0}^{1}\left[t\left(f^{r}(a)-f^{r}(b)\right)+f^{r}(b)\right]^{\frac{1}{r}}[g(a)]^{t}[g(b)]^{1-t} d t .
\end{aligned}
$$

Again, Cauchy's inequality shows that

$$
\begin{aligned}
& \int_{0}^{1}\left[t\left(f^{r}(a)-f^{r}(b)\right)+f^{r}(b)\right]^{\frac{1}{r}}[g(a)]^{t}[g(b)]^{1-t} d t \\
& \quad \leq \frac{1}{2} \int_{0}^{1}\left[t\left(f^{r}(a)-f^{r}(b)\right)+f^{r}(b)\right]^{\frac{2}{r}} d t+\frac{1}{2} \int_{0}^{1}[g(a)]^{2 t}[g(b)]^{2-2 t} d t .
\end{aligned}
$$

We have

$$
\int_{0}^{1}\left[t\left(f^{r}(a)-f^{r}(b)\right)+f^{r}(b)\right]^{\frac{2}{r}} d t=\frac{r}{r+2}\left(\frac{f^{r+2}(b)-f^{r+2}(a)}{f^{r}(b)-f^{r}(a)}\right) .
$$

Similar to the proof of Theorem 2.10, we can show that

$$
\int_{0}^{1}[g(a)]^{2 t}[g(b)]^{2-2 t} d t=\frac{1}{2}\left[g(b)^{2}-g(a)^{2}\right] \ln \frac{g(b)}{g(a)} .
$$

Using (10), (11) and (12), we can obtain the desired result. 


\section{Competing interests}

The authors declare that they have no competing interests.

\section{Authors' contributions}

All authors contributed equally to the manuscript and read and approved the final draft.

\section{Author details}

${ }^{1}$ Department of Mathematics, Faculty of Mathematical Science and Computer, Tarbiat Moallem University, 599 Taleghani Avenue, Tehran, 15618, Iran. ${ }^{2}$ Department of Mathematics, Garmsar Branch, Islamic Azad University, Garmsar, Iran.

${ }^{3}$ Department of Mathematics and Institute for Mathematical Research, University Putra Malaysia, UPM, Serdang, Selangor 43400, Malaysia.

\section{Acknowledgements}

The authors express their sincere thanks to the referee(s) for the careful and detailed reading of the manuscript and very helpful suggestions that improved the manuscript substantially. The third author also acknowledges that this project was partially supported by University Putra Malaysia.

\section{Received: 14 March 2012 Accepted: 13 September 2012 Published: 2 October 2012}

\section{References}

1. Hadamard, J: Étude sur les proprietes des fonctions entieres en particulier d'une fonction considérée par Riemann. J. Math. Pures Appl. 58, 171-215 (1893)

2. Dragomir, SS, Pearce, CEM: Selected Topics on Hermite-Hadamard Inequalities and Its Applications. RGMIA Monograph (2002)

3. Dragomir, SS: Refinements of the Hermite-Hadamard integral inequality for log-convex functions. Aust. Math. Soc. Gaz. 28(3), 129-134 (2001)

4. Mihaly, B: Hermite-Hadamard-type inequalities for generalized convex functions. J. Inequal. Pure Appl. Math. 9(3), Article ID 63 (2008) (PhD thesis)

5. Zabandan, G: A new refinement of the Hermite-Hadamard inequality for convex functions. J. Inequal. Pure Appl. Math. 10(2), Article ID 45 (2009)

6. Pearce, CEM, Pečcaric, J, Šimić, V: Stolarsky means and Hadamard's inequality. J. Math. Anal. Appl. 220, 99-109 (1998)

7. Ngoc, NPN, Vinh, NV, Hien, PTT: Integral inequalities of Hadamard type for $r$-convex functions. Int. Math. Forum 4(35), 1723-1728 (2009)

8. Yang, GS: Refinementes of Hadamard inequality for $r$-convex functions. Indian J. Pure Appl. Math. 32(10), 1571-1579 (2001)

9. Bessenyei, M: Hermite-Hadamard-type inequalities for generalized 3-convex functions. Publ. Math. (Debr.) 65(1-2), 223-232 (2004)

doi:10.1186/1029-242X-2012-215

Cite this article as: Zabandan et al.: The Hermite-Hadamard inequality for $r$-convex functions. Journal of Inequalities and Applications 2012 2012:215

\section{Submit your manuscript to a SpringerOpen ${ }^{\circ}$ journal and benefit from:}

- Convenient online submission

- Rigorous peer review

- Immediate publication on acceptance

- Open access: articles freely available online

- High visibility within the field

- Retaining the copyright to your article 